\title{
Robust stabilisation of 2D state-delayed stochastic systems with randomly occurring uncertainties and nonlinearities
}

\author{
Zhaoxia Duan ${ }^{\mathrm{a}}$, Zhengrong Xiang ${ }^{\mathrm{a}, *}$ and Hamid Reza Karimi ${ }^{\mathrm{b}}$ \\ ${ }^{a}$ School of Automation, Nanjing University of Science and Technology, Nanjing, People's Republic of China; ${ }^{b}$ Department of \\ Engineering, Faculty of Engineering and Science, University of Agder, Grimstad, Norway
}

(Received 5 April 2013; accepted 25 July 2013)

\begin{abstract}
This paper is concerned with the state feedback control problem for a class of two-dimensional (2D) discrete-time stochastic systems with time-delays, randomly occurring uncertainties and nonlinearities. Both the sector-like nonlinearities and the norm-bounded uncertainties enter into the system in random ways, and such randomly occurring uncertainties and nonlinearities obey certain mutually uncorrelated Bernoulli random binary distribution laws. Sufficient computationally tractable linear matrix inequality-based conditions are established for the $2 \mathrm{D}$ nonlinear stochastic time-delay systems to be asymptotically stable in the mean-square sense, and then the explicit expression of the desired controller gains is derived. An illustrative example is provided to show the usefulness and effectiveness of the proposed method.
\end{abstract}

Keywords: 2D stochastic systems; randomly occurring uncertainties; randomly occurring nonlinearities; linear matrix inequality (LMI)

\section{Introduction}

As many practical systems can be modelled as twodimensional (2D) systems, such as those in image data processing and transmission, gas absorption, thermal processes and water stream heating (Kaczorek 1985), the analysis and synthesis of 2D systems have received considerable attention, for example, 2D state-space realisation theory was researched in Fornasini and Marchesini (1976), Hinamoto (1997) and Bisiacco (1995) studied the stability and 2D optimal control theory, Sebek (1993) and Du and Xie (2002) addressed the $H_{\infty}$ control problem for effectively solving the noise attenuation problem for 2D systems.

Due to the increasing complexity of large-scale systems, it is crucial to model the system complexity in an appropriate way to facilitate the system analysis and design. It is well known that time-delay and stochastic disturbance are two of the most important factors constituting the complexity that should be taken into account in system modelling. In the past few decades, a great number of results on the stability analysis of delayed systems have been proposed (He, Wang, Lin and Wu 2007). For 2D systems, many literatures have investigated the problems of delay-dependent stability and stabilisation (Paszke, Lam, Galkowski, Xu and Lin 2004; Xu and Lam 2005; Xu, Lam and Zou 2005; Xu, Zou, Lu and Xu 2005; Xu and Yu 2006; Chen 2010). Stochastic one-dimensional (1D) systems with and without delay have received a great deal of research attention, and some problems have been studied (see Gao and
Ahmed 1986, 1987; Dragan, Halanay and Morozan 1992; Bouhtouri, Hinrichsen and Pritchard 1999). Recently, some results on stochastic 2D systems have been obtained. For example, the analysis and synthesis of 2D stochastic systems with system matrices subject to stochastic perturbation were researched (Gao, Lam, Wang, and Xu 2004; Gao, Lam, Xu and Wang 2005). Yao and Wang (2010) made effort on the robust stabilisation of 2D state-delays systems with stochastic perturbation. Liang, Wang and Liu (2012) investigated the robust state estimation problem for a class of 2D systems with state delays and stochastic disturbances.

On the other hand, uncertainty and nonlinearity are another two key factors constituting the complexity of the practical systems and should also be considered in sys-tem modelling. Recently, the filtering and control prob-lems for 1D systems with stochastic nonlinearities have been investigated (Wang, Yang and Liu 2006; Yang, Wang and Ho 2006; Wang, Liu and Liu 2008; Wang, Liu, Guo and Liu 2010; Liu, Wang and Wang 2011). 2D systems with nonlinearities and uncertainties have been studied by many researchers (Shen, Wang, Shu, and Wei 2010; Pakshin, Galkowski and Rogers 2011; Dhawan 2012; Huang and Xiang 2013; Wei, Wang, and Shen 2013). How-ever, some practical systems may be influenced by additive nonlinear disturbances and uncertainties that are caused by environmental circumstances such as sudden environment changes, modification of the operating point of a linearised 
model of nonlinear systems, etc. In other words, the nonlinear disturbances and uncertainties may occur in a probabilistic way and are randomly changeable in terms of their types or intensity. This kind of uncertainty and nonlinearity can be referred to as randomly occurring uncertainty and randomly occurring nonlinearity, respectively. For 1D systems, the randomly occurring nonlinearities and randomly occurring uncertainties have recently received some interest in the literature. For example, Wang, Wang and Liang (2009) investigated the synchronisation problem for delayed complex networks with randomly occurring nonlinearities and multiple stochastic disturbances. Hu, Wang, Gao and Stergioulas (2012) considered the robust sliding mode control of discrete stochastic systems with randomly occurring uncertainties and nonlinearities. This class of systems with incomplete information such as incomplete measurements and probabilistic time-varying delay gradually becomes a hot research topic among researchers (Wan and Fang 2013; Zhang, Wang, Li and Fei 2013). Unfortunately, to the best of the authors' knowledge, the corresponding results for $2 \mathrm{D}$ systems have been very few. More specifically, the robust stabilisation problem for 2D state-delayed systems with randomly occurring nonlinearities and uncertainties has not yet been investigated to date, and the purpose of this paper is therefore to fill in such a gap.

Motivated by the above observations, in this paper, we aim to deal with the robust stabilisation problem for a class of 2D state-delayed systems with stochastic perturbation, randomly occurring nonlinearities and uncertainties. The main contribution of this paper is mainly threefold. (1) The 2D system under consideration is general enough to include time-delays, randomly occurring nonlinearities, randomly occurring uncertainties and stochastic disturbances. (2) An energy-like quadratic Lyapunov function is developed, together with the intensive use of stochastic analysis and sector-bounded nonlinearity condition, to establish the easy-to-verify conditions in form of linear matrix inequality (LMI) under which the addressed 2D system achieves robust asymptotical stability in the mean-square sense. (3) A state feedback controller is designed to ensure the robust asymptotical stability of the corresponding closed-loop system.

The remainder of this paper is organised as follows. Section 2 formulates the problem and presents some preliminary results. The analysis of robust asymptotical stability is given in Section 3. The robust state feedback controller design is developed in Section 4. An example is given to illustrate the effectiveness of the approach in Section 5. Finally, the paper is concluded in Section 6.

Notation: The notations used throughout the paper are fairly standard except where otherwise stated. $R^{n}$ and $R^{m \times n}$ denote, respectively, the $n$-dimensional Euclidean space and the set of all real matrices of dimension $m \times n$. $I$ and 0 represent the identity matrix and the zero matrix, respectively, with appropriate dimensions. The notation $X \geq Y$ $(X>Y)$ means that $X-Y$ is positive semi-definite (positive definite). The superscript ' $T$ ' stands for matrix transposition and ' $*$ ' in a matrix is used to denote the term that is induced by symmetry. $\operatorname{diag}\{\cdots\}$ means a block-diagonal matrix and $\|\cdot\|$ refers to the Euclidean norm. The set of all non-negative integers is denoted by $Z_{+}$. Moreover, let $E\{x\}$ and $E\{x \mid y\}$ stand, respectively, the expectation of stochastic variable $x$ and the expectation of $x$ conditional on $y$ with respect to the given probability measurement.

\section{Preliminary and problem formulation}

Consider the following 2D discrete state-delayed systems with randomly occurring uncertainties, randomly occurring nonlinearities and stochastic disturbance:

$$
\begin{aligned}
{\left[\begin{array}{c}
x^{h}(k+1, l) \\
x^{v}(k, l+1)
\end{array}\right]=} & (A+\alpha(k, l) \Delta A(k, l))\left[\begin{array}{c}
x^{h}(k, l) \\
x^{v}(k, l)
\end{array}\right] \\
& +\left(A_{d}+\beta(k, l) \Delta A_{d}(k, l)\right) \\
& \times\left[\begin{array}{c}
x^{h}(k-d(k, l), l) \\
x^{v}(k, l-d(k, l))
\end{array}\right]+B u(k, l) \\
& +\delta(k, l) C_{1} f\left(\left[\begin{array}{c}
x^{h}(k, l) \\
x^{v}(k, l)
\end{array}\right]\right) \\
& +(1-\delta(k, l)) C_{2} g\left(\left[\begin{array}{c}
x^{h}(k, l) \\
x^{v}(k, l)
\end{array}\right]\right) \\
& +\left((D+\Delta D)\left[\begin{array}{c}
x^{h}(k, l) \\
x^{v}(k, l)
\end{array}\right]\right. \\
& \left.+\left(D_{d}+\Delta D_{d}\right)\left[\begin{array}{c}
x^{h}(k-d(k, l), l) \\
x^{v}(k, l-d(k, l))
\end{array}\right]\right) \\
& \times w(k, l),
\end{aligned}
$$

where $x^{h}(k, l)$ is the horizontal state in $R^{n_{1}}, x^{v}(k, l)$ is the vertical state in $R^{n_{2}}, x(k, l)$ is the whole state in $R^{N}$ with $N=n_{1}+n_{2}$, and $k, l$ are the non-negative integers, $u(k, l)$ is the control input. $z(k, l)$ is the controlled output, $d(k, l)$ denotes the time-delay satisfying $d_{1} \leq d(k, l) \leq d_{2}, A, A_{d}$, $B, C_{1}, C_{2}, D$ and $D_{d}$ are known real constant matrices with compatible dimensions, $f(\cdot) \in R^{N}$ and $g(\cdot) \in R^{N}$ are nonlinear functions, $w(k, l)$ is a standard random scalar signal defined on $(\Omega, F$, Prob) satisfying

$$
\begin{aligned}
& E\{w(k, l) w(i, j)\}=1, \quad(k, l)=(i, j), \\
& E\{w(k, l) w(i, j)\}=0,(k, l) \neq(i, j)
\end{aligned}
$$

Let $\left(\Omega, F,\left\{F_{p}\right\}_{p \in z_{+}}\right.$, Prob) be a filtered probability space where $\left\{F_{p}\right\}_{p \in z_{+}}$is the family of sub $\sigma$-algebras of $F$ generated by $\{w(k, l)\}_{k, l \in Z_{+}}$. Specially, $F_{p}$ is assumed to be the minimal $\sigma$-algebras generated by $\{w(k, l)\}_{0 \leq k+l \leq p-1}$, while $F_{0}$ is assumed to be some given sub $\sigma$-algebras of $F$ and independent of $F_{p}$ for all $p>0$. The initial boundary 
condition associated with system (1) is given by

$$
\begin{array}{lrrr}
x^{h}(k, l)=h(k, l), & \forall 0 \leq l \leq z_{1}, & -d_{2} \leq k \leq 0 \\
x^{h}(k, l)=0, & \forall l>z_{1}, & -d_{2} \leq k \leq 0 \\
x^{v}(k, l)=v(k, l), & \forall 0 \leq k \leq z_{2}, & -d_{2} \leq l \leq 0 \\
x^{v}(k, l)=0, & \forall k>z_{2}, & -d_{2} \leq l \leq 0 \\
h(0,0) & =v(0,0), &
\end{array}
$$

where $z_{1}<\infty$ and $z_{2}<\infty$ are positive integers, and $h(k, l) \in R_{+}^{n_{1}}$ and $v(k, l) \in R_{+}^{n_{2}}$ are given vectors belonging to $F_{0}$.

Remark 1: Note that the initial condition (3) has appeared in the literature (Yao and Wang 2010). The requirements of $z_{1}<\infty$ and $z_{2}<\infty$ are necessary for the state trajectories to have some convergence property $x(k, l) \rightarrow 0$ as $k+l$ $\rightarrow$

0 . In other words, one has to consider only bounded initial conditions, otherwise one may have unbounded trajectories, because the convergence property implies in particular that $x^{h}(0, l) \rightarrow 0$ as $l \rightarrow \infty$ and $x^{v}(k, 0) \rightarrow$ 0 as $k \rightarrow \infty$.

Remark 2: From an application point of view, the Equation (1) can be used to describe the relation between voltage and current in a long transmission line, some processes of gas absorption, water stream heating, air drying, and some thermal processes, for example in chemical reactors, heat exchangers and pipe furnaces.

$\delta(k, l)$ is a stochastic variable that describes the follow-ing random events for system (1):

Event 1: system (1) experiences the nonlinear function $f(\cdot)$.

Event 2: system (1) experiences the nonlinear function $g(\cdot)$.

Let $\delta(k, l)$ be a Bernoulli distributed sequence defined by

$$
\delta(k, l)=\left\{\begin{array}{l}
1: \text { if Event } 1 \text { occurs } \\
0: \text { if Event } 2 \text { occurs. }
\end{array}\right.
$$

It follows that $\delta(k, l)$ satisfies

$$
\begin{aligned}
& \operatorname{Prob}\{\delta(k, l)=1\}=E\{\delta(k, l)\}=\bar{\delta}, \\
& \operatorname{Prob}\{\delta(k, l)=0\}=1-E\{\delta(k, l)\}=1-\bar{\delta},
\end{aligned}
$$

where the constant $\bar{\delta} \in[0,1]$ reflects the occurrence probability of the event of the nonlinear functions $f(\cdot)$ and $g(\cdot)$.

Remark 3: In this paper, the random variable $\delta(k, l)$ is used to model the probability distribution of the nonlinear functions. To our knowledge, this represents the first attempt to take into account the occurrence of different nonlinear functions in a probabilistic way for the addressed 2D discrete systems. In other words, in system (1), the two terms $\delta(k, l) C_{1} f(\cdot)$ and $(1-\delta(k, l)) C_{2} g(\cdot)$ can be used to account for the binary switch between these two nonlinear functions according to the given probability distribution.

The stochastic variables $\alpha(k, l) \in R$ and $\beta(k, l) \in R$ are also Bernoulli distributed white sequences taking the values of either zero or one with

$$
\begin{array}{ll}
\operatorname{Prob}\{\alpha(k, l)=1\}=\bar{\alpha}, & \operatorname{Prob}\{\alpha(k, l)=0\}=1-\bar{\alpha}, \\
\operatorname{Prob}\{\beta(k, l)=1\}=\bar{\beta}, & \operatorname{Prob}\{\beta(k, l)=0\}=1-\bar{\beta},
\end{array}
$$

where $\bar{\alpha} \in[0,1]$ and $\bar{\beta} \in[0,1]$ are known constants.

Remark 4: In this paper, $\alpha(k, l)$ and $\beta(k, l)$ are used to model the randomly occurring uncertainties that lead to incomplete information process problem and are commonly encountered in various engineering systems.

The unknown real-valued matrices $\Delta A(k, l)$, $\Delta A_{d}(k, l), \quad \Delta D(k, l)$ and $\Delta D_{d}(k, l)$ represent the norm-bounded parameter uncertainties of the following structure:

$$
\left[\Delta A \Delta A_{d} \Delta D \Delta D_{d}\right]=M F(k, l)\left[\begin{array}{llll}
N_{1} & N_{2} & N_{3} & N_{4}
\end{array}\right],
$$

where $M, N_{1}, N_{2}, N_{3}$ and $N_{4}$ are known constant matrices and $F(k, l)$ is an unknown matrix function with Lebesgue measurable elements satisfying

$$
F^{T}(k, l) F(k, l) \leq I .
$$

Assumption 1: The boundary condition is independent of $w(i, j)$ and is assumed to be

$$
\lim _{N \rightarrow \infty} E\left\{\sum_{l=-d_{2}}^{0} \sum_{k=1}^{N}\left(\left\|x^{h}(l, k)\right\|^{2}+\left\|x^{v}(k, l)\right\|^{2}\right)\right\}<\infty .
$$

Assumption 2: For $\forall x, y \in R^{N}$, the nonlinear functions $f(\cdot)$ and $g(\cdot)$ satisfy the following sector-bounded conditions, respectively:

$$
\begin{aligned}
& {\left[f(x)-f(y)-X_{1}(x-y)\right]^{T}[f(x)-f(y)} \\
& \left.\quad-X_{2}(x-y)\right] \leq 0, \\
& {\left[g(x)-g(y)-Y_{1}(x-y)\right]^{T}[g(x)-g(y)} \\
& \left.\quad-Y_{2}(x-y)\right] \leq 0,
\end{aligned}
$$

where $X_{1}, Y_{1}$ and $X_{2}, Y_{2}$ are real constant matrices with $X_{2}-X_{1} \geq 0$ and $Y_{2}-Y_{1} \geq 0$. 
Remark 5: The nonlinear functions $f(\cdot)$ and $g(\cdot)$ satisfying Assumption 2 are said to belong to the sector $\left[X_{1}, X_{2}\right]$ and $\left[Y_{1}, Y_{2}\right]$, respectively. Note that the sectorbounded nonlinearity of stochastic systems has been studied by Wang et al. (2008) and Wang, Wang and Liang (2008). It should be pointed out that such a nonlinear condition is more general than the usual Lipschitz conditions that have been widely used in Yu, Cao and Lü (2008) and Liang, Wang and Liu (2008). The sector-bounded nonlinearity of 2D systems has also been considered in Liang et al. (2012).

Assumption 3: (a) the stochastic variables $\alpha(k, l), \beta(k$, $l)$ and $\delta(k, l)$ are mutually uncorrelated random variables that are also unrelated with $w(k, l)$. (b) the stochastic variables $\alpha(k, l), \beta(k, l)$ and $\delta(k, l)$ are independent of F.

Lemma 1 (Xie 1996): Let $U, V, W$ and $X$ be real matrices of appropriate dimensions with $X$ satisfying $X=$ $X^{T}$, then for all $V^{T} V \leq I$, the inequality $X+U V W+W$ ${ }^{T} V^{T} U^{T}<0$ holds, if and only if there exists a positive scalar $\varepsilon$ such that $X+\varepsilon U U^{T}+\varepsilon^{-1} W^{T} W<0$.

Definition 1: The 2D discrete stochastic system with delay in (1) with Assumptions 1-3 is said to be mean-square asymptotically stable if the following equality

$$
\lim _{k+l \rightarrow \infty} E\left\{\|x(k, l)\|^{2}\right\}=0
$$

holds for any the zero input $u(k, l)=0$ and initial condition (4) satisfying (8), where $x(k, l)=\left[\begin{array}{c}x^{h}(k, l) \\ x^{v}(k, l)\end{array}\right]$.

The problem to be addressed in this paper is to develop a technique of robust stabilisation for $2 \mathrm{D}$ discrete state-delayed systems with randomly occurring uncertainties, randomly occurring nonlinearities and stochastic disturbance. More specifically, we are concerned with the following robust stabilisation problem: Determine a state feedback controller

$$
u(k, l)=K\left[\begin{array}{l}
x^{h}(k, l) \\
x^{v}(k, l)
\end{array}\right]
$$

for system (1) such that the resulting closed-loop system is robustly mean-square asymptotically stable. In this case, the system (1) is said to be robustly stochastically stabilisable.

\section{Robust stability analysis}

In this section, an LMI approach will be developed to analyse the robust stability of the problem formulated in the previous section.

Consider system (1) with zero control input $u(k, l)=0$, that is

$$
\begin{aligned}
x^{+}(k, l)= & (A+\alpha(k, l) \Delta A(k, l)) x(k, l)+\left(A_{d}\right. \\
& \left.+\beta(k, l) \Delta A_{d}(k, l)\right) x_{d}(k, l)+\delta(k, l) \\
& \times C_{1} f(x(k, l))+(1-\delta(k, l)) C_{2} g(x(k, l)) \\
& +\left((D+\Delta D) x(k, l)+\left(D_{d}+\Delta D_{d}\right) x_{d}(k, l)\right) \\
& \times w(k, l),
\end{aligned}
$$

where

$$
\begin{aligned}
& x^{+}(k, l)=\left[\begin{array}{c}
x^{h}(k+1, l) \\
x^{v}(k, l+1)
\end{array}\right], \quad x(k, l)=\left[\begin{array}{c}
x^{h}(k, l) \\
x^{v}(k, l)
\end{array}\right], \\
& x_{d}(k, l)=\left[\begin{array}{c}
x^{h}(k-d(k, l), l) \\
x^{v}(k, l-d(k, l))
\end{array}\right] .
\end{aligned}
$$

Theorem 1: System (13) with the boundary condition (3) satisfying (8) is mean-square asymptotically stable if there exist matrices $\bar{P}=\operatorname{diag}\left\{\bar{P}^{h}, \bar{P}^{v}\right\}>0, \bar{Q}=$ $\operatorname{diag}\left\{\bar{Q}^{h}, \bar{Q}^{v}\right\}>0$, and positive scalars $\varepsilon_{1}, \varepsilon_{2}, \varepsilon_{3}$ and $\varepsilon_{4}$ such that the following LMI holds

$$
\left[\begin{array}{ll}
S_{1} & S_{2} \\
* & S_{3}
\end{array}\right]<0
$$

where

$$
S_{1}=\left[\begin{array}{ccccccccc}
\tilde{\Omega} & 0 & -\bar{P} X^{T} & -\bar{P} Y^{T} & \bar{P} \Gamma_{1} & \bar{P} D^{T} & 0 & \bar{P} \Upsilon_{1} & \bar{P} \Upsilon_{2} \\
* & -\bar{Q} & 0 & 0 & \bar{P} A_{d}^{T} & \bar{P} D_{d}^{T} & 0 & 0 & 0 \\
* & * & -2 I & 0 & \bar{\delta} C_{1}^{T} & 0 & 0 & 0 & 0 \\
* & * & * & -2 I & (1-\bar{\delta}) C_{2}^{T} & 0 & 0 & 0 & 0 \\
* & * & * & * & -\bar{P}+\varepsilon_{1} M M^{T} & 0 & 0 & 0 & 0 \\
* & * & * & * & * & -\bar{P}+\varepsilon_{2} M M^{T} & 0 & 0 & 0 \\
* & * & * & * & * & * & -\bar{P}+\varepsilon_{3} M M^{T} & 0 & 0 \\
* & * & * & * & * & * & * & -\bar{P} & 0 \\
* & * & * & * & * & * & * & * & -\bar{P}
\end{array}\right]
$$




$$
S_{2}=\left[\begin{array}{ccccccc}
0 & 0 & 0 & \bar{\alpha} \bar{P} N_{1}^{T} & \bar{P} N_{3}^{T} & \sqrt{\bar{\alpha}(1-\bar{\alpha})} \bar{P} N_{1}^{T} & 0 \\
0 & 0 & 0 & \bar{\beta} \bar{P} N_{1}^{T} & \bar{P} N_{4}^{T} & 0 & \sqrt{\bar{\beta}(1-\bar{\beta})} \bar{P} N_{2}^{T} \\
0 & \Upsilon_{3} & 0 & 0 & 0 & 0 & 0 \\
0 & 0 & \Upsilon_{4} & 0 & 0 & 0 & 0 \\
0 & 0 & 0 & 0 & 0 & 0 & 0 \\
0 & 0 & 0 & 0 & 0 & 0 & 0 \\
0 & 0 & 0 & 0 & 0 & 0 & 0 \\
0 & 0 & 0 & 0 & 0 & 0 & 0 \\
0 & 0 & 0 & 0 & 0 & 0 & 0
\end{array}\right]
$$

$$
S_{3}=\operatorname{diag}\left\{-\bar{P}+\varepsilon_{4} M M^{T},-\bar{P},-\bar{P},-\varepsilon_{1} I,-\varepsilon_{2} I,\right.
$$$$
\left.-\varepsilon_{3} I,-\varepsilon_{4} I\right\} \text {, }
$$

where

$$
\begin{aligned}
\Gamma_{1} & =A^{T}+\bar{\delta} X_{1}^{T} C_{1}^{T}+(1-\bar{\delta}) Y_{1}^{T} C_{2}^{T}, \\
\tilde{\Omega} & =-\bar{P}+\left(d_{2}-d_{1}+1\right) \bar{Q}, \\
\Upsilon_{1} & =\sqrt{\bar{\delta}(1-\bar{\delta})} X_{1}^{T} C_{1}^{T}, \quad \Upsilon_{2}=\sqrt{\bar{\delta}(1-\bar{\delta})} Y_{1}^{T} C_{2}^{T}, \\
\Upsilon_{3} & =\sqrt{\bar{\delta}(1-\bar{\delta})} C_{1}^{T}, \quad \Upsilon_{4}=\sqrt{\bar{\delta}(1-\bar{\delta})} C_{2}^{T}, \\
X & =X_{2}-X_{1}, Y=Y_{2}-Y_{1} .
\end{aligned}
$$

Proof: We decompose the nonlinear functions $f(x)$ and $g(x)$ into linear and nonlinear parts as

$$
f(x)=f_{n}(x)+X_{1} x, g(x)=g_{n}(x)+Y_{1} x,
$$

where the nonlinearities $f_{n}(x)$ and $g_{n}(x)$ belong to the set $\Phi_{1}$ and $\Phi_{2}$, respectively, given by

$$
\begin{aligned}
& \Phi_{1}=\left\{f_{n}(x) \mid f_{n}(x)^{T}\left(f_{n}(x)-X x\right) \leq 0\right\}, \\
& \Phi_{2}=\left\{g_{n}(x) \mid g_{n}(x)^{T}\left(g_{n}(x)-Y x\right) \leq 0\right\} .
\end{aligned}
$$

Since $f(x(k, l))$ and $g(x(k, l))$ belong to sectors $\left[X_{1}, X_{2}\right]$ and $\left[Y_{1}, Y_{2}\right]$, respectively, the system (13) becomes

$$
\begin{aligned}
x^{+}(k, l)= & \left(A+\alpha(k, l) \Delta A(k, l)+\delta(k, l) C_{1} X_{1}\right. \\
& \left.+(1-\delta(k, l)) C_{2} Y_{1}\right) x(k, l)+\left(A_{d}+\beta(k, l)\right. \\
& \left.\times \Delta A_{d}(k, l)\right) x_{d}(k, l)+\delta(k, l) C_{1} f_{n}(x(k, l)) \\
& +(1-\delta(k, l)) C_{2} g_{n}(x(k, l))+((D+\Delta D) \\
& \left.\times x(k, l)+\left(D_{d}+\Delta D_{d}\right) x_{d}(k, l)\right) w(k, l)
\end{aligned}
$$

Then we choose a Lyapunov functional candidate for system (17) as follows:

$$
V(k, l)=V^{h}(k, l)+V^{v}(k, l)
$$

with

$$
\begin{aligned}
& V^{h}(k, l)=V_{1}^{h}(k, l)+V_{2}^{h}(k, l)+V_{3}^{h}(k, l), \\
& V^{v}(k, l)=V_{1}^{v}(k, l)+V_{2}^{v}(k, l)+V_{3}^{v}(k, l),
\end{aligned}
$$

where

$$
\begin{aligned}
& V_{1}^{h}(k, l)=x^{h T}(k, l) P^{h} x^{h}(k, l), \\
& V_{1}^{v}(k, l)=x^{v T}(k, l) P^{v} x^{v}(k, l), \\
& V_{2}^{h}(k, l)=\sum_{r=-d(k, l)}^{-1} x^{h T}(k+r, l) Q^{h} x^{h}(k+r, l), \\
& V_{2}^{v}(k, l)=\sum_{t=-d(k, l)}^{-1} x^{v T}(k, l+t) Q^{v} x^{v}(k, l+t), \\
& V_{3}^{h}(k, l)=\sum_{m=-d_{2}+2}^{-d_{1}+1} \sum_{r=m-1}^{-1} x^{h T}(k+r, l) Q^{h} x^{h}(k+r, l), \\
& V_{3}^{v}(k, l)=\sum_{m=-d_{2}+2}^{-d_{1}+1} \sum_{t=m-1}^{-1} x^{v T}(k, l+t) Q^{v} x^{v}(k, l+t) .
\end{aligned}
$$

Then we have

$$
\begin{aligned}
& E\left\{V^{+}(k, l) \mid x(k, l)\right\}-V(k, l) \\
&= \sum_{i=1}^{3}\left[E\left\{V_{i}^{h}(k+1, l) \mid x(k, l)\right\}\right. \\
&\left.\quad-V_{i}^{h}(k, l)+E\left\{V_{i}^{v}(k, l+1) \mid x(k, l)\right\}-V_{i}^{v}(k, l)\right],
\end{aligned}
$$

where $V^{+}(k, l)=V^{h}(k+1, l)+V^{v}(k, l+1)$. Moreover, the following equations hold for the terms in the right-hand side of the Equation (19):

$$
\begin{aligned}
E\{ & \left.V_{1}^{h}(k+1, l) \mid x(k, l)\right\}-V_{1}^{h}(k, l) \\
= & E\left\{x^{h T}(k+1, l) P^{h} x^{h}(k+1, l) \mid x(k, l)\right\} \\
& -x^{h T}(k, l) P^{h} x^{h}(k, l) .
\end{aligned}
$$


$E\left\{V_{1}^{v}(k, l+1) \mid x(k, l)\right\}-V_{1}^{v}(k, l)$

$$
=E\left\{x^{v T}(k, l+1) P^{v} x^{v}(k, l+1) \mid x(k, l)\right\}
$$$$
-x^{v T}(k, l) P^{v} x^{v}(k, l) \text {. }
$$

$$
E\left\{V_{2}^{h}(k+1, l) \mid x(k, l)\right\}-V_{2}^{h}(k, l)
$$

$$
\begin{aligned}
&= E\left\{\sum_{r=-d(k+1, l)}^{-1} x^{h T}(k+r+1, l) Q^{h} x^{h}(k+r+1, l)\right. \\
&\mid x(k, l)\}-\sum_{r=-d(k, l)}^{-1} x^{h T}(k+r, l) Q^{h} x^{h}(k+r, l) \\
&=E\left\{\sum_{r=-d(k+1, l)}^{-d_{1}-1} x^{h T}(k+r+1, l) Q^{h} x^{h}(k+r+1, l)\right. \\
&+\sum_{r=-d_{1}}^{-1} x^{h T}(k+r+1, l) Q^{h} x^{h}(k+r+1, l) \\
&-\sum_{r=-d(k, l)}^{-d_{1}} x^{h T}(k+r, l) Q^{h} x^{h}(k+r+1, l) \\
&\left.-\sum_{r=-d_{1}+1}^{-1} x^{h T}(k+r, l) Q^{h} x^{h}(k+r, l) \mid x(k, l)\right\} \\
&= E\left\{\sum_{r=-d(k+1, l)+1}^{-d_{1}} x^{h T}(k+r, l) Q^{h} x^{h}(k+r, l)\right. \\
&+\sum_{r=-d_{1}+1}^{-1} x^{h T}(k+r, l) Q^{h} x^{h}(k+r, l)+x^{h T}(k, l) \\
& \times Q^{h} x^{h}(k, l)-x^{h T}(k-d(k, l), l) Q^{h} x^{h}(k-d(k, l), l) \\
&\left.-\sum_{r=-d(k, l)+1}^{-1} x^{h T}(k+r, l) Q^{h} x^{h}(k+r, l) \mid x(k, l)\right\} .
\end{aligned}
$$

$E\left\{V_{2}^{v}(k, l+1) \mid x(k, l)\right\}-V_{2}^{v}(k, l)$

$$
\begin{aligned}
= & E\left\{\sum_{t=-d(k, l+1)}^{-1} x^{v T}(k, l+t+1) Q^{v} x^{v}(k, l+t+1)\right. \\
& \mid x(k, l)\}-\sum_{t=-d(k, l)}^{-1} x^{v T}(k, l+t) Q^{v} x^{v}(k, l+t) \\
= & E\left\{\sum_{t=-d(k, l+1)}^{-d_{1}-1} x^{v T}(k, l+t+1) Q^{v} x^{v}(k, l+t+1)\right. \\
& +\sum_{t=-d_{1}}^{-1} x^{v T}(k, l+t+1) Q^{v} x^{v}(k, l+t+1) \\
& -\sum_{t=-d(k, l)}^{-d_{1}} x^{v T}(k, l+t) Q^{v} x^{v}(k, l+t) \\
& \left.-\sum_{t=-d_{1}+1}^{-1} x^{v T}(k, l+t) Q^{v} x^{v}(k, l+t) \mid x(k, l)\right\}
\end{aligned}
$$

$$
\begin{aligned}
= & E\left\{\sum_{t=-d(k, l+1)+1}^{-d_{1}} x^{v T}(k, l+t) Q^{v} x^{v}(k, l+t)\right. \\
& +\sum_{t=-d_{1}+1}^{-1} x^{v T}(k, l+t) Q^{v} x^{v}(k, l+t)+x^{v T}(k, l) \\
& \times Q^{v} x^{v}(k, l)-x^{v T}(k, l-d(k, l)) Q^{v} x^{v}(k, l-d(k, l)) \\
& \left.-\sum_{t=-d(k, l)+1}^{-1} x^{v T}(k, l+t) Q^{v} x^{v}(k, l+t) \mid x(k, l)\right\} .
\end{aligned}
$$

$$
\begin{aligned}
E & \left\{V_{3}^{h}(k+1, l) \mid x(k, l)\right\}-V_{3}^{h}(k, l) \\
= & E\left\{\sum_{m=-d_{2}+2}^{-d_{1}+1} \sum_{r=m-1}^{-1} x^{h T}(k+r+1, l) Q^{h} x^{h}(k+r+1, l)\right. \\
& \left.-\sum_{m=-d_{2}+2}^{-d_{1}+1} \sum_{r=m-1}^{-1} x^{h T}(k+r, l) Q^{h} x^{h}(k+r, l) \mid x(k, l)\right\} \\
= & E\left\{\left(d_{2}-d_{1}\right) x^{h T}(k, l) Q^{h} x^{h}(k, l)\right. \\
& \left.\quad-\sum_{m=-d_{2}+1}^{-d_{1}} x^{h T}(k+m, l) Q^{h} x^{h}(k+m, l) \mid x(k, l)\right\} .
\end{aligned}
$$

$E\left\{V_{3}^{v}(k, l+1) \mid x(k, l)\right\}-V_{3}^{v}(k, l)$

$=E\left\{\sum_{m=-d_{2}+2}^{-d_{1}+1} \sum_{t=m-1}^{-1} x^{v T}(k, l+t+1) Q^{v} x^{v}(k, l+t+1)\right.$ $\left.-\sum_{m=-d_{2}+2}^{-d_{1}+1} \sum_{t=m-1}^{-1} x^{v T}(k, l+t) Q^{v} x^{v}(k, l+t) \mid x(k, l)\right\}$ $=E\left\{\left(d_{2}-d_{1}\right) x^{v T}(k, l) Q^{v} x^{v}(k, l)\right.$

$$
\left.-\sum_{m=-d_{2}+1}^{-d_{1}} x^{v T}(k, l+m) Q^{v} x^{v}(k, l+m) \mid x(k, l)\right\} .
$$

Substituting (20)-(25) into (19) and along the trajectory of system (17), one can obtain

$$
\begin{aligned}
E & \left\{V^{+}(k, l) \mid x(k, l)\right\}-V(k, l) \\
= & E\left\{x^{h T}(k+1, l) P^{h} x^{h}(k+1, l)-x^{h T}(k, l) P^{h} x^{h}(k, l)\right. \\
& +x^{v T}(k, l+1) P^{v} x^{v}(k, l+1)-x^{v T}(k, l) P^{v} x^{v}(k, l) \\
& +x^{h T}(k, l) Q^{h} x^{h}(k, l)+x^{v T}(k, l) Q^{v} x^{v}(k, l) \\
& -x^{h T}(k-d(k, l), l) Q^{h} x^{h}(k-d(k, l), l) \\
& -x^{v T}(k, l-d(k, l)) Q^{v} x^{v}(k, l-d(k, l))
\end{aligned}
$$




$$
\begin{aligned}
& +\left(d_{2}-d_{1}\right) x^{h T}(k, l) Q^{h} x^{h}(k, l)+\left(d_{2}-d_{1}\right) x^{v T}(k, l) \\
& \times Q^{v} x^{v}(k, l)+\sum_{r=-d_{1}+1}^{-1} x^{h T}(k+r, l) Q^{h} x^{h}(k+r, l) \\
& -\sum_{r=-d(k, l)+1}^{-1} x^{h T}(k+r, l) Q^{h} x^{h}(k+r, l) \\
& +\sum_{t=-d_{1}+1}^{-1} x^{v T}(k, l+t) Q^{v} x^{v}(k, l+t) \\
& -\sum_{t=-d(k, l)+1}^{-1} x^{v T}(k, l+t) Q^{v} x^{v}(k, l+t) \\
& +\sum_{r=-d(k+1, l)+1}^{-d_{1}} x^{h T}(k+r, l) Q^{h} x^{h}(k+r, l) \\
& -\sum_{m=-d_{2}+1}^{-d_{1}} x^{h T}(k+m, l) Q^{h} x^{h}(k+m, l) \\
& +\sum_{t=-d(k, l+1)+1}^{-d_{1}} x^{v T}(k, l+t) Q^{v} x^{v}(k, l+t) \\
& \left.-\sum_{m=-d_{2}+1}^{-d_{1}} x^{v T}(k, l+m) Q^{v} x^{v}(k, l+m) \mid x(k, l)\right\} .
\end{aligned}
$$

Since $d_{1} \leq d(k, l) \leq d_{2}$, we have

$$
\begin{aligned}
& \sum_{r=-d_{1}+1}^{-1} x^{h T}(k+r, l) Q^{h} x^{h}(k+r, l) \\
& \leq \sum_{r=-d(k, l)+1}^{-1} x^{h T}(k+r, l) Q^{h} x^{h}(k+r, l), \\
& \sum_{t=-d_{1}+1}^{-1} x^{v T}(k, l+t) Q^{v} x^{v}(k, l+t) \\
& \leq \sum_{t=-d(k, l)+1}^{-1} x^{v T}(k, l+t) Q^{v} x^{v}(k, l+t), \\
& \sum_{r=-d(k+1, l)+1}^{-d_{1}} x^{h T}(k+r, l) Q^{h} x^{h}(k+r, l) \\
& \leq \sum_{m=-d_{2}+1}^{-d_{1}} x^{h T}(k+m, l) Q^{h} x^{h}(k+m, l), \\
& \sum_{t=-d(k, l+1)+1}^{-d_{1}} x^{v T}(k, l+t) Q^{v} x^{v}(k, l+t) \\
& \leq \sum_{m=-d_{2}+1}^{-d_{1}} x^{v T}(k, l+m) Q^{v} x^{v}(k, l+m) .
\end{aligned}
$$

Then from (27)-(30), (19) can be rewritten as

$$
\begin{aligned}
& E\left\{V^{+}(k, l) \mid x(k, l)\right\}-V(k, l) \\
& \quad \leq E\left\{x^{+T}(k, l) P x^{+}(k, l)-x^{T}(k, l) P x(k, l)\right. \\
& \quad-x_{d}^{T}(k, l) Q x_{d}(k, l)+\left(d_{2}-d_{1}+1\right) x^{T}(k, l) Q x(k, l) \\
& \quad \mid x(k, l)\},
\end{aligned}
$$

where

$$
P=\operatorname{diag}\left\{P_{h}, P_{v}\right\}, Q=\operatorname{diag}\left\{Q_{h}, Q_{v}\right\} .
$$

For simplicity, we take

$$
\begin{aligned}
\Delta A & =\Delta A(k, l), \Delta A_{d}=\Delta A_{d}(k, l), \\
\Delta D & =\Delta D(k, l), \Delta D_{d}=\Delta D_{d}(k, l), \alpha=\alpha(k, l), \\
\beta & =\beta(k, l), \delta=\delta(k, l) .
\end{aligned}
$$

Then system (17) can be represented as follows:

$$
\begin{aligned}
x^{+}(k, l)= & \left(A+\bar{\alpha} \Delta A+\bar{\delta} C_{1} X_{1}+(1-\bar{\delta}) C_{2} Y_{1}\right) x(k, l) \\
& +\left(A_{d}+\bar{\beta} \Delta A_{d}\right) x_{d}(k, l)+\bar{\delta} C_{1} f_{n}(x(k, l)) \\
& +(1-\bar{\delta}) C_{2} g_{n}(x(k, l))+((\alpha-\bar{\alpha}) \Delta A \\
& \left.+(\delta-\bar{\delta}) C_{1} X_{1}+(\bar{\delta}-\delta) C_{2} Y_{1}\right) x(k, l) \\
& +(\beta-\bar{\beta}) \Delta A_{d} x_{d}(k, l)+(\delta-\bar{\delta}) C_{1} f_{n}(x(k, l)) \\
& +(\bar{\delta}-\delta) C_{2} g_{n}(x(k, l))+((D+\Delta D) x(k, l) \\
& \left.+\left(D_{d}+\Delta D_{d}\right) x_{d}(k, l)\right) w(k, l) .
\end{aligned}
$$

Applying (32) into (31), one can obtain

$$
\begin{aligned}
E\left\{V^{+}\right. & (k, l) \mid x(k, l)\}-V(k, l) \\
\leq & E\left\{\left[\left(A+\bar{\alpha} \Delta A+\bar{\delta} C_{1} X_{1}+(1-\bar{\delta}) C_{2} Y_{1}\right) x(k, l)\right.\right. \\
& +\bar{\delta} C_{1} f_{n}(x(k, l))+\left(A_{d}+\bar{\beta} \Delta A_{d}\right) x_{d}(k, l) \\
& \left.+(1-\bar{\delta}) C_{2} g_{n}(x(k, l))\right]^{T} P\left[\left(A+\bar{\alpha} \Delta A+\bar{\delta} C_{1} X_{1}\right.\right. \\
& \left.+(1-\bar{\delta}) C_{2} Y_{1}\right) x(k, l)+\bar{\delta} C_{1} f_{n}(x(k, l)) \\
& \left.+\left(A_{d}+\bar{\beta} \Delta A_{d}\right) x_{d}(k, l)+(1-\bar{\delta}) C_{2} g_{n}(x(k, l))\right] \\
& +\left[\left((\alpha-\bar{\alpha}) \Delta A+(\delta-\bar{\delta}) C_{1} X_{1}+(\bar{\delta}-\delta) C_{2} Y_{1}\right) x(k, l)\right. \\
& +(\beta-\bar{\beta}) \Delta A_{d} x_{d}(k, l)+(\delta-\bar{\delta}) C_{1} f_{n}(x(k, l)) \\
& \left.+(\bar{\delta}-\delta) C_{2} g_{n}(x(k, l))\right]^{T} P\left[(\beta-\bar{\beta}) \Delta A_{d} x_{d}(k, l)\right. \\
& \left((\alpha-\bar{\alpha}) \Delta A+(\delta-\bar{\delta}) C_{1} X_{1}+(\bar{\delta}-\delta) C_{2} Y_{1}\right) x(k, l) \\
& \left.+(\delta-\bar{\delta}) C_{1} f_{n}(x(k, l))+(\bar{\delta}-\delta) C_{2} g_{n}(x(k, l))\right] \\
& +\left((D+\Delta D) x(k, l)+\left(D_{d}+\Delta D_{d}\right) x_{d}(k, l)\right)^{T} \\
& \times P\left((D+\Delta D) x(k, l)+\left(D_{d}+\Delta D_{d}\right) x_{d}(k, l)\right) \\
& +x^{T}(k, l) P x(k, l)-x_{d}^{T}(k, l) Q x_{d}(k, l) \\
& \left.+\left(d_{2}-d_{1}+1\right) x^{T}(k, l) Q x(k, l)\right\} .
\end{aligned}
$$

From (16), we get that the following inequality holds:

$$
\begin{aligned}
& E\left\{V^{+}(k, l) \mid x(k, l)\right\}-V(k, l) \\
& \quad \leq E\left\{V^{+}(k, l) \mid x(k, l)\right\}-V(k, l)-f_{n}(x)^{T}\left(f_{n}(x)\right. \\
& \quad-X x)-g_{n}(x)^{T}\left(g_{n}(x)-Y x\right) .
\end{aligned}
$$


Noting (33) and (34), we have

$$
\begin{aligned}
& E\left\{V^{+}(k, l) \mid x(k, l)\right\}-V(k, l)=\left[\begin{array}{c}
x(k, l) \\
x_{d}(k, l) \\
f_{n}(x(k, l)) \\
g_{n}(x(k, l))
\end{array}\right]^{T}\left\{\left[\begin{array}{c}
\Gamma_{1}+\bar{\alpha} \Delta A^{T} \\
A_{d}^{T}+\bar{\beta} \Delta A_{d}^{T} \\
\bar{\delta} C_{1}^{T} \\
(1-\bar{\delta}) C_{2}^{T}
\end{array}\right] P\left[\begin{array}{c}
\Gamma_{1}+\bar{\alpha} \Delta A^{T} \\
A_{d}^{T}+\bar{\beta} \Delta A_{d}^{T} \\
\bar{\delta} C_{1}^{T} \\
(1-\bar{\delta}) C_{2}^{T}
\end{array}\right]^{T}+\left[\begin{array}{c}
D^{T}+\Delta D^{T} \\
D_{d}^{T}+\Delta D_{d}^{T} \\
0 \\
0
\end{array}\right]\right. \\
& \left.\times P\left[\begin{array}{c}
D^{T}+\Delta D^{T} \\
D_{d}^{T}+\Delta D_{d}^{T} \\
0 \\
0
\end{array}\right]^{T}+\left[\begin{array}{cccc}
\Omega_{1} & 0 & -X^{T} & -Y^{T} \\
* & \Omega_{2} & 0 & 0 \\
* & * & \Omega_{3} & 0 \\
* & * & * & \Omega_{4}
\end{array}\right]\right\}\left[\begin{array}{c}
x(k, l) \\
x_{d}(k, l) \\
f_{n}(x(k, l)) \\
g_{n}(x(k, l))
\end{array}\right]
\end{aligned}
$$

where

$$
\begin{aligned}
& \Gamma_{1}=A^{T}+\bar{\delta} X_{1}^{T} C_{1}^{T}+(1-\bar{\delta}) Y_{1}^{T} C_{2}^{T}, \\
& \Omega_{1}=-P+\left(d_{2}-d_{1}+1\right) Q+\bar{\alpha}(1-\bar{\alpha}) \Delta A^{T} P \Delta A+\bar{\delta}(1-\bar{\delta}) X_{1}^{T} C_{1}^{T} P C_{1} X_{1}+\bar{\delta}(1-\bar{\delta}) Y_{1}^{T} C_{2}^{T} P C_{2} Y_{1}, \\
& \Omega_{2}=-Q+\bar{\beta}(1-\bar{\beta}) \Delta A_{d}^{T} P \Delta A_{d}, \\
& \Omega_{3}=\bar{\delta}(1-\bar{\delta}) C_{1}^{T} P C_{1}-2 I, \\
& \Omega_{4}=\bar{\delta}(1-\bar{\delta}) C_{2}^{T} P C_{2}-2 I .
\end{aligned}
$$

\begin{tabular}{|c|c|c|c|c|c|c|c|c|c|c|c|c|}
\hline$-\bar{\Omega}$ & 0 & $-X^{T}$ & $-Y^{T}$ & $\Gamma_{1}+\bar{\alpha} \Delta A^{T}$ & $D^{T}+\Delta D^{T}$ & $\overline{\bar{\chi}(1-\bar{\alpha})} \Delta A^{T}$ & $\Upsilon_{1}$ & $\Upsilon_{2}$ & 0 & 0 & 0 & \multirow{12}{*}{$<0$} \\
\hline$*$ & $-Q$ & 0 & 0 & $A_{d}^{T}+\bar{\beta} \Delta A_{d}^{T}$ & $D_{d}^{T}+\Delta D_{d}^{T}$ & 0 & 0 & 0 & $\sqrt{\bar{\beta}(1-\bar{\beta})} \Delta A_{d}^{T}$ & 0 & 0 & \\
\hline$*$ & $*$ & $-2 I$ & 0 & $\bar{\delta} C_{1}^{T}$ & 0 & 0 & 0 & 0 & 0 & $\Upsilon_{3}$ & 0 & \\
\hline * & $*$ & $*$ & $-2 I$ & $(1-\bar{\delta}) C_{2}^{T}$ & 0 & 0 & 0 & 0 & 0 & 0 & $\Upsilon_{4}$ & \\
\hline * & $*$ & $*$ & $*$ & $-P^{-1}$ & 0 & 0 & 0 & 0 & 0 & 0 & 0 & \\
\hline * & $*$ & $*$ & * & $*$ & $-P^{-1}$ & 0 & 0 & 0 & 0 & 0 & 0 & \\
\hline * & $*$ & $*$ & $*$ & $*$ & * & $-P^{-1}$ & 0 & 0 & 0 & 0 & 0 & \\
\hline * & $*$ & $*$ & $*$ & $*$ & $*$ & $*$ & $-P^{-1}$ & 0 & 0 & 0 & 0 & \\
\hline * & $*$ & $*$ & * & * & * & * & $*$ & $-P^{-1}$ & 0 & 0 & 0 & \\
\hline * & $*$ & $*$ & $*$ & $*$ & * & * & $*$ & $*$ & $-P^{-1}$ & 0 & 0 & \\
\hline$\uparrow$ & $*$ & $*$ & $*$ & $*$ & * & * & * & $*$ & $*$ & $-P^{-1}$ & 0 & \\
\hline . & $*$ & $*$ & $*$ & $*$ & $*$ & $*$ & $*$ & * & $*$ & * & $-P^{-1}$ & \\
\hline
\end{tabular}

By Schur complement, we can get that $E\left\{V^{+}(k, l) \mid x(k, l)\right\}-V(k, l)<0$ if the following inequality holds

From (6), we have

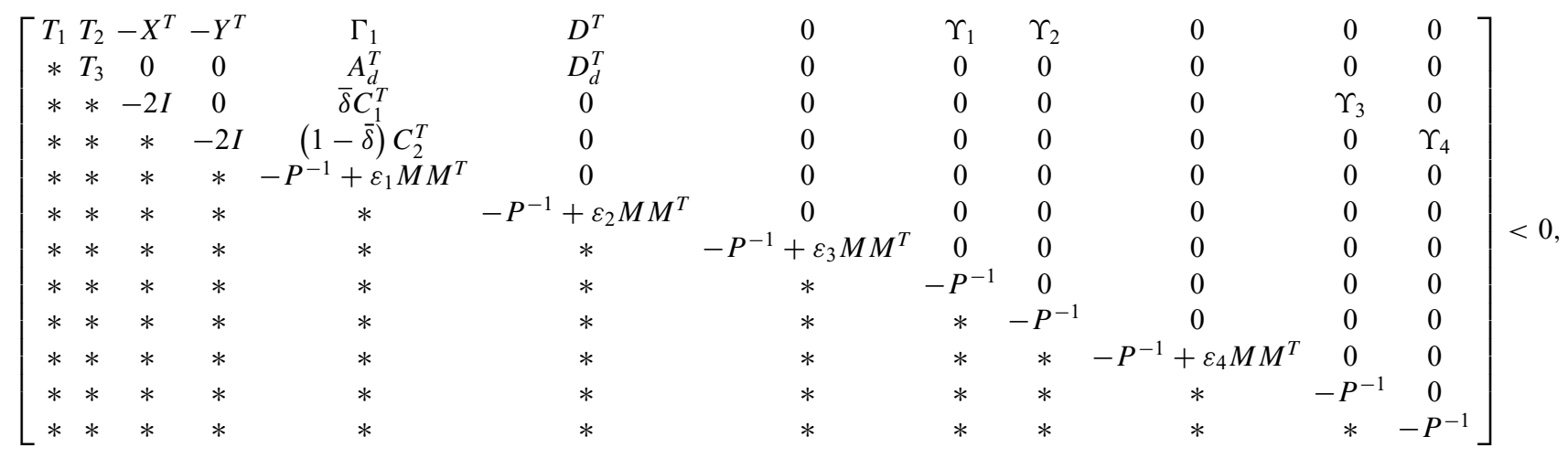


where

$$
\begin{aligned}
& T_{1}=\bar{\Omega}+\left(\varepsilon_{1}^{-1} \bar{\alpha}^{2}+\varepsilon_{3}^{-1} \bar{\alpha}(1-\bar{\alpha})\right) N_{1}^{T} N_{1}+\varepsilon_{2}^{-1} N_{3}^{T} N_{3}, \\
& T_{2}=\varepsilon_{1}^{-1} \bar{\alpha} \bar{\beta} N_{1}^{T} N_{2}+\varepsilon_{2}^{-1} N_{3}^{T} N_{4}, \\
& T_{3}=-Q+\left(\varepsilon_{1}^{-1} \bar{\beta}^{2}+\varepsilon_{4}^{-1} \bar{\beta}(1-\bar{\beta})\right) N_{2}^{T} N_{2}+\varepsilon_{2}^{-1} N_{4}^{T} N_{4} .
\end{aligned}
$$

Using Schur complement, we can get

$$
\left[\begin{array}{cc}
\Sigma_{1} & \Sigma_{2} \\
* & \Sigma_{3}
\end{array}\right]<0
$$

Note that for any nonnegative integer $Z>\max \left(z_{1}, z_{2}\right)$, it holds that $V^{h}(0, Z)=0$ and $V^{v}(Z, 0)=0$. Then summing up both sides of (40) from $Z-1$ to 0 with respect to $l$ and 0 to $Z-1$ with respect to $k$ leads to

$$
\begin{aligned}
& \sum_{k=0}^{Z} \sum_{l=0}^{Z} E\left\{V^{h}(k+1, l)-V^{h}(k, l)+V^{v}(k, l+1)-V^{v}(k, l)\right\} \\
& \quad=\sum_{l=0}^{Z} E\left\{V^{h}(Z, l)-V^{h}(0, l)\right\}+\sum_{k=0}^{Z} E\left\{V^{v}(k, Z)-V^{v}(k, 0)\right\} \\
& \quad \leq-\rho \sum_{k=0}^{Z} \sum_{l=0}^{Z} E\left\{\|x(k, l)\|^{2}\right\}
\end{aligned}
$$

where

$$
\begin{aligned}
& \Sigma_{1}=\left[\begin{array}{ccccccccc}
\bar{\Omega} & 0 & -X^{T} & -Y^{T} & \Gamma_{1} & D^{T} & 0 & \Upsilon_{1} & \Upsilon_{2} \\
* & -Q & 0 & 0 & A_{d}^{T} & D_{d}^{T} & 0 & 0 & 0 \\
* & * & -2 I & 0 & \bar{\delta} C_{1}^{T} & 0 & 0 & 0 & 0 \\
* & * & * & -2 I & (1-\bar{\delta}) C_{2}^{T} & 0 & 0 & 0 & 0 \\
* & * & * & * & -P^{-1}+\varepsilon_{1} M M^{T} & 0 & 0 & 0 & 0 \\
* & * & * & * & * & -P^{-1}+\varepsilon_{2} M M^{T} & 0 & 0 & 0 \\
* & * & * & * & * & * & -P^{-1}+\varepsilon_{3} M M^{T} & 0 & 0 \\
* & * & * & * & * & * & * & -P^{-1} & 0 \\
* & * & * & * & * & * & * & * & -P^{-1}
\end{array}\right], \\
& {\left[\begin{array}{ccccccc}
0 & 0 & 0 & \bar{\alpha} N_{1}^{T} & N_{3}^{T} & \sqrt{\bar{\alpha}(1-\bar{\alpha})} N_{1}^{T} & 0 \\
0 & 0 & 0 & \bar{\beta} N_{1}^{T} & N_{4}^{T} & 0 & \sqrt{\bar{\beta}(1-\bar{\beta})} N_{2}^{T} \\
0 & \Upsilon_{3} & 0 & 0 & 0 & 0 & 0
\end{array}\right]} \\
& \Sigma_{2}=\left[\begin{array}{ccccccc}
0 & \Upsilon_{3} & 0 & 0 & 0 & 0 & 0 \\
0 & 0 & \Upsilon_{4} & 0 & 0 & 0 & 0 \\
0 & 0 & 0 & 0 & 0 & 0 & 0 \\
0 & 0 & 0 & 0 & 0 & 0 & 0 \\
0 & 0 & 0 & 0 & 0 & 0 & 0 \\
0 & 0 & 0 & 0 & 0 & 0 & 0 \\
0 & 0 & 0 & 0 & 0 & 0 & 0
\end{array}\right], \\
& \Sigma_{3}=\operatorname{diag}\left\{-P^{-1}+\varepsilon_{4} M M^{T},-P^{-1},-P^{-1},-\varepsilon_{1} I,-\varepsilon_{2} I,-\varepsilon_{3} I,-\varepsilon_{4} I\right\} .
\end{aligned}
$$

Pre- and post-multiplying (38) by $\operatorname{diag}\left\{P^{-1}, P^{-1}\right.$, $\underbrace{I, \ldots, I}_{14}\}$, and let $\bar{P}=P^{-1}$ and $\bar{Q}=P^{-1} Q P^{-1}$, we can get the LMI (14) from (38), which means that there exists a scalar $\rho$ such that

$$
\begin{aligned}
E & \left\{V^{+}(k, l) \mid x(k, l)\right\}-V(k, l) \\
& \left.<\left[\begin{array}{c}
x(k, l) \\
x_{d}(k, l) \\
f(x(k, l)) \\
g(x(k, l))
\end{array}\right]^{T}\left[\begin{array}{cccc}
-\rho I & 0 & 0 & 0 \\
* & 0 & 0 & 0 \\
* & * & 0 & 0 \\
* & * & * & 0
\end{array}\right]\right\}\left[\begin{array}{c}
x(k, l) \\
x_{d}(k, l) \\
f(x(k, l)) \\
g(x(k, l))
\end{array}\right] \\
& =-\rho\|x(k, l)\|^{2} .
\end{aligned}
$$

Taking mathematical expectation on both sides of (39) gives

$$
\begin{aligned}
& E\left\{V^{h}(k+1, l)-V^{h}(k, l)+V^{v}(k, l+1)-V^{v}(k, l)\right\} \\
& \quad<-\rho E\left\{\|x(k, l)\|^{2}\right\} .
\end{aligned}
$$

and, therefore,

$$
\begin{aligned}
\sum_{k=0}^{Z} \sum_{l=0}^{Z} E\left\{\|x(k, l)\|^{2}\right\} \leq & \frac{1}{\rho}\left\{\sum_{l=0}^{Z} E\left\{V^{h}(0, l)-V^{h}(Z, l)\right\}\right. \\
& \left.+\sum_{k=0}^{Z} E\left\{V^{v}(k, 0)-V^{v}(k, Z)\right\}\right\} \\
& \leq \frac{1}{\rho} \sum_{l=0}^{Z} E\left\{V^{h}(0, l)+V^{v}(0, l)\right\} .
\end{aligned}
$$

It follows from Assumption 1 and (17) that $\sum_{k=0}^{Z} \sum_{l=0}^{Z} E\left\{\|x(k, l)\|^{2}\right\}<\infty$ which implies that

$$
\lim _{k+l \rightarrow \infty} E\left\{\|x(k, l)\|^{2}\right\}=0 .
$$


Then from Definition 1, we can conclude that system (13) is asymptotically stable in the mean-square sense, the proof of Theorem 1 is completed.

\section{Robust stabilisation}

Based on Theorem 1, we can design a state feedback controller in the form of (12) for system (1) to ensure that and $\varepsilon_{4}$ such that the following LMI holds:

$$
\left[\begin{array}{cc}
\Lambda_{1} & \Lambda_{2} \\
* & \Lambda_{3}
\end{array}\right]<0,
$$

where

$$
\begin{aligned}
& \Lambda_{1}=\left[\begin{array}{ccccccccc}
\tilde{\Omega} & 0 & -\bar{P} X^{T} & -\bar{P} Y^{T} & \bar{P} \tilde{\Gamma}+K_{c}^{T} B^{T} & \bar{P} D^{T} & 0 & \bar{P} \Upsilon_{1} & \bar{P} \Upsilon_{2} \\
* & -\bar{Q} & 0 & 0 & \bar{P} A_{d}^{T} & \bar{P} D_{d}^{T} & 0 & 0 & 0 \\
* & * & -2 I & 0 & \bar{\delta} C_{1}^{T} & 0 & 0 & 0 & 0 \\
* & * & * & -2 I & (1-\bar{\delta}) C_{2}^{T} & 0 & 0 & 0 & 0 \\
* & * & * & * & -\bar{P}+\varepsilon_{1} M M^{T} & 0 & 0 & 0 & 0 \\
* & * & * & * & * & -\bar{P}+\varepsilon_{2} M M^{T} & 0 & 0 & 0 \\
* & * & * & * & * & * & -\bar{P}+\varepsilon_{3} M M^{T} & 0 & 0 \\
* & * & * & * & * & * & * & -\bar{P} & 0 \\
* & * & * & * & * & * & * & * & -\bar{P}
\end{array}\right],
\end{aligned}
$$

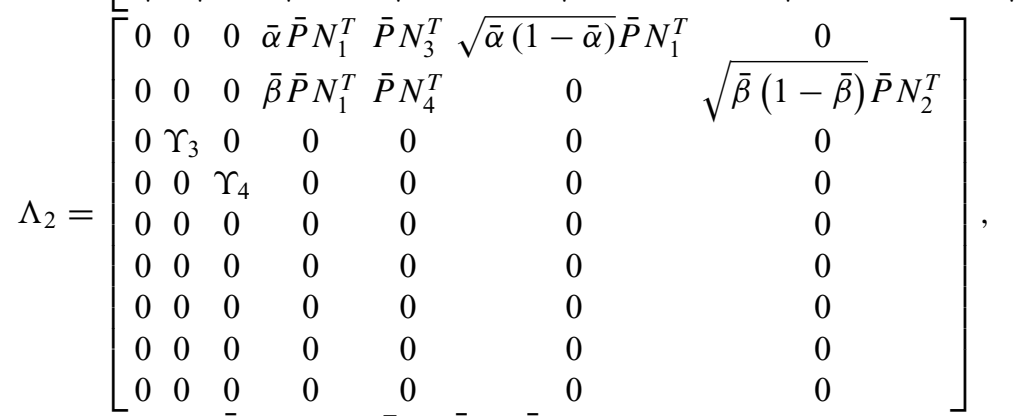

$$
\begin{aligned}
& \Lambda_{3}=\operatorname{diag}\left\{-\bar{P}+\varepsilon_{4} M M^{T},-\bar{P},-\bar{P},-\varepsilon_{1} I,-\varepsilon_{2} I,-\varepsilon_{3} I,-\varepsilon_{4} I\right\},
\end{aligned}
$$

the closed-loop system is robustly mean-square asymptotically stable. Under controller (12), the corresponding closed-loop system for system (1) can be written as

$$
\begin{aligned}
x^{+}(k, l)= & \left(A+B K+\alpha(k, l) \Delta A(k, l)+\delta(k, l) C_{1} X_{1}\right. \\
& \left.+(1-\delta(k, l)) C_{2} Y_{1}\right) x(k, l)+\left(A_{d}+\beta(k, l)\right. \\
& \left.\times \Delta A_{d}(k, l)\right) x_{d}(k, l)+\delta(k, l) C_{1} f_{n}(x(k, l)) \\
& +(1-\delta(k, l)) C_{2} g_{n}(x(k, l)) \\
& +\left((D+\Delta D) x(k, l)+\left(D_{d}\right.\right. \\
& \left.\left.+\Delta D_{d}\right) x_{d}(k, l)\right) w(k, l),
\end{aligned}
$$

where

$$
\begin{aligned}
& x^{+}(k, l)=\left[\begin{array}{l}
x^{h}(k+1, l) \\
x^{v}(k, l+1)
\end{array}\right], x(k, l)=\left[\begin{array}{l}
x^{h}(k, l) \\
x^{v}(k, l)
\end{array}\right], \\
& x_{d}(k, l)=\left[\begin{array}{l}
x^{h}(k-d(k, l), l) \\
x^{v}(k, l-d(k, l))
\end{array}\right] .
\end{aligned}
$$

Theorem 2: The system in (1) is robustly stochastically stabilisable if there exist matrices $\bar{P}=\operatorname{diag}\left\{\bar{P}^{h}, \bar{P}^{v}\right\}>0$, $\bar{Q}=\operatorname{diag}\left\{\bar{Q}^{h}, \bar{Q}^{v}\right\}>0, K_{c}$ and positive scalars $\varepsilon_{1}, \varepsilon_{2}, \varepsilon_{3}$ where

$$
\begin{aligned}
\tilde{\Omega} & =-\bar{P}+\left(d_{2}-d_{1}+1\right) \bar{Q}, \\
\tilde{\Gamma} & =A^{T}+\bar{\delta} X_{1}^{T} C_{1}^{T}+(1-\bar{\delta}) Y_{1}^{T} C_{2}^{T}, \\
\Upsilon_{1} & =\sqrt{\bar{\delta}(1-\bar{\delta})} X_{1}^{T} C_{1}^{T}, \Upsilon_{2}=\sqrt{\bar{\delta}(1-\bar{\delta})} Y_{1}^{T} C_{2}^{T}, \\
\Upsilon_{3} & =\sqrt{\bar{\delta}(1-\bar{\delta})} C_{1}^{T}, \Upsilon_{4}=\sqrt{\bar{\delta}(1-\bar{\delta})} C_{2}^{T}, \\
X & =X_{2}-X_{1}, Y=Y_{2}-Y_{1} .
\end{aligned}
$$

In this case, the desired control gain in (12) is given by

$$
K=K_{c} \bar{P}^{-1} .
$$

Proof: Substituting $A$ with $A+B K$ in (14) and letting $K_{c}=K \bar{P}$, we can get the LMI condition (45). By Theorem 1 , we conclude that the closed-loop system (44) is asymptotically stable. This completes the proof.

Remark 6: The condition (45) in Theorem 2 is given in terms of LMI which can be easy-verified by using the LMI toolbox of Matlab. Then by (46), we can compute the value of the state feedback controller. The feasibility of the proposed method will be illustrated by the example given in the next section. 


\section{Illustrative example}

In this section, we present an example to illustrate the effectiveness of the proposed approach.

Consider the Darboux equation with delays that can be used to describe some process of gas absorption, water stream heating and air drying (Kaczorek 1985):

$$
\begin{aligned}
\frac{\partial^{2} s(x, t)}{\partial x \partial t}= & a_{1} \frac{\partial s(x, t)}{\partial t}+a_{2} \frac{\partial s(x, t)}{\partial x}+a_{0} s(x, t) \\
& +a_{d} s(x, t-\tau)+b u(x, t),
\end{aligned}
$$

where $s(x, t)$ is an unknown function at $x \in\left[0, x_{f}\right]$ (space) and $t \in\left[0, t_{f}\right]$ (time), $a_{0}, a_{1}, a_{2}, a_{d}, b$ are real coefficients, $u(x, t)$ is a given input function.

Let us define

$$
r(x, t)=\frac{\partial s(x, t)}{\partial t}-a_{2} s(x, t) .
$$

By (48) we can transform (47) into an equivalent system with first order differential equations of the form.

$$
\begin{aligned}
{\left[\begin{array}{l}
\frac{\partial r(x, t)}{\partial x} \\
\frac{\partial s(x, t)}{\partial t}
\end{array}\right]=} & {\left[\begin{array}{cc}
a_{1} & a_{1} a_{2}+a_{0} \\
1 & a_{2}
\end{array}\right]\left[\begin{array}{l}
r(x, t) \\
s(x, t)
\end{array}\right] } \\
& +\left[\begin{array}{c}
a_{d} \\
0
\end{array}\right] s(x, t-\tau)+\left[\begin{array}{l}
b \\
0
\end{array}\right] u(x, t(49)
\end{aligned}
$$

From (47), we can get

$$
\begin{aligned}
r(0, t) & =\left.\frac{\partial s(x, t)}{\partial}\right|_{x=0}-a_{2} s(0, t) \\
& =\frac{d s(0, t)}{d t}-a_{2} s(0, t)=R(t) .
\end{aligned}
$$

Taking $\quad r(k, l)=r(k \Delta x, l \Delta t) \quad$ and $\quad s(k, l)=$ $s(k \Delta x, l \Delta t)$, then

$$
\begin{aligned}
& \frac{\partial r(x, t)}{\partial x}=\frac{r(k+1, l)-r(k, l)}{\Delta x}, \\
& \frac{\partial s(x, t)}{\partial t} \stackrel{\simeq}{=} \frac{s(k, l+1)-s(k, l)}{\Delta t} .
\end{aligned}
$$

We can obtain from (49)

$$
\begin{aligned}
{\left[\begin{array}{c}
r(k+1, l) \\
s(k, l+1)
\end{array}\right]=} & {\left[\begin{array}{cc}
1+a_{1} \Delta x & \left(a_{1} a_{2}+a_{0}\right) \Delta x \\
\Delta t & 1+a_{2} \Delta t
\end{array}\right]\left[\begin{array}{c}
r(k, l) \\
s(k, l)
\end{array}\right] } \\
& +\left[\begin{array}{c}
a_{d} \Delta x \\
0
\end{array}\right] s(k, l-d(l)) \\
& +\left[\begin{array}{c}
b \Delta x \\
0
\end{array}\right] u(k, l) .
\end{aligned}
$$

Due to environmental circumstances such as sudden environment changes, the practical systems may be influenced by additive stochastic disturbance, randomly occurring uncertainties and nonlinearities, that is, the uncertainties and nonlinearities may occur in a probabilistic way. In this example, we assume the sector-like nonlinearities and the norm-bounded uncertainties enter into the system in random ways, and such randomly occurring uncertainties and nonlinearities obey certain mutually uncorrelated Bernoulli random binary distribution laws. Let $x^{h}(k, l)=r(k, l)$, $x^{v}(k, l)=s(k, l)$, and the nonlinear function and noise intensity function vector be given by

$$
\begin{aligned}
f & (x(k, l)) \\
& =\left[\begin{array}{c}
0.4 \tan \left(-x^{h}(k, l)\right)+0.2 x^{h}(k, l)+0.1 x^{v}(k, l) \\
0.1 x^{h}(k, l)-0.4 \tan \left(x^{v}(k, l)\right)+0.2 x^{v}(k, l)
\end{array}\right], \\
g & (x(k, l)) \\
& =\left[\begin{array}{c}
0.1 x^{h}(k, l)-0.4 \tan \left(x^{v}(k, l)\right)+0.2 x^{v}(k, l) \\
0.4 \tan \left(-x^{h}(k, l)\right)+0.2 x^{h}(k, l)+0.1 x^{v}(k, l)
\end{array}\right] .
\end{aligned}
$$

Then we can get a 2D discrete state-delayed system in the form of (1) with parameters as follows:

$$
\begin{aligned}
A= & {\left[\begin{array}{ll}
-0.2400 & 0.1307 \\
-0.1440 & 0.0008
\end{array}\right], A_{d}=\left[\begin{array}{cc}
0 & 0.1089 \\
0 & 0
\end{array}\right], } \\
B= & {\left[\begin{array}{cc}
1 & -0.3 \\
0 & 0
\end{array}\right], C_{1}=\left[\begin{array}{cc}
-0.06 & 0.04 \\
0.03 & -0.05
\end{array}\right], } \\
C_{2}= & {\left[\begin{array}{cc}
-0.02 & 0.03 \\
-0.08 & -0.12
\end{array}\right], D=\left[\begin{array}{cc}
0 & 0.1 \\
0.2 & 0.1
\end{array}\right], } \\
D_{d}= & {\left[\begin{array}{cc}
0 & 0.02 \\
0 & 0
\end{array}\right] M=\left[\begin{array}{l}
0.02 \\
0.01
\end{array}\right], N_{1}=\left[\begin{array}{ll}
0.01 & 0.02
\end{array}\right], } \\
N_{2}= & {\left[\begin{array}{ll}
0.01 & 0.02
\end{array}\right], N_{3}=\left[\begin{array}{ll}
0.02 & 0.05
\end{array}\right], N_{4}=\left[\begin{array}{ll}
0.04 & 0.02
\end{array}\right], } \\
\bar{\delta}= & 0.54, \bar{\alpha}=0.78, \bar{\beta}=0.77, \\
& d(k, l)=2.5+0.5 \sin \left(\frac{\pi}{2} l\right) .
\end{aligned}
$$

It is easy to verify that

$$
\begin{aligned}
& X_{1}=\left[\begin{array}{cc}
-0.3 & 0.1 \\
0.1 & -0.3
\end{array}\right], X_{2}=\left[\begin{array}{ll}
0.2 & 0.1 \\
0.1 & 0.2
\end{array}\right], \\
& Y_{1}=\left[\begin{array}{cc}
0.1 & -0.3 \\
-0.3 & 0.1
\end{array}\right], Y_{2}=\left[\begin{array}{ll}
0.1 & 0.2 \\
0.2 & 0.1
\end{array}\right], d_{1}=2, d_{2}=3 .
\end{aligned}
$$

By using the LMI toolbox of Matlab, the solution of (43) is given as

$$
\begin{aligned}
\bar{P} & =\left[\begin{array}{cc}
1.7281 & 0 \\
0 & 1.7372
\end{array}\right], \bar{Q}=\left[\begin{array}{cc}
0.3166 & 0 \\
0 & 0.3202
\end{array}\right], \\
K_{c} & =\left[\begin{array}{ll}
0.4179 & 10.0595 \\
0.0498 & 34.2561
\end{array}\right], \varepsilon_{1}=1.8709, \\
\varepsilon_{2} & =1.4062, \varepsilon_{3}=1.7059, \quad \varepsilon_{4}=1.6839 .
\end{aligned}
$$






Figure 1. State trajectory of $x^{h}(k, l)$.

By Theorem 2, the desired state feedback controller can be constructed as

$$
u(k, l)=\left[\begin{array}{ll}
-0.8358 & -20.1189 \\
-0.0995 & -68.5122
\end{array}\right] x(k, l)
$$

The boundary conditions are given by

$$
\begin{gathered}
x^{h}(i, j)=0.2, \forall 0 \leq j \leq 15, i=0 \\
x^{v}(i, j)=0.2, \forall 0 \leq i \leq 15,-d_{2} \leq j \leq 0 .
\end{gathered}
$$

The state responses of the system are shown in Figures 1 and 2, which demonstrate the closed-loop system is asymptotically stable in the mean-square sense.



Figure 2. State trajectory of $x^{v}(k, l)$.

\section{Conclusion}

The 2D stochastic time-delay systems with randomly occurring uncertainties and randomly occurring nonlinearities is first considered in this paper and the corresponding robust stability and stabilisation problems have been studied. Both the sector-like nonlinearities and the norm-bounded uncertainties enter into the system in random ways, and they obey certain mutually uncorrelated Bernoulli distributed white sequences. An effective LMI approach has been proposed to design a state feedback controller such that, for all admissible nonlinearities and uncertainties, the overall closed-loop system is asymptotically stable in the meansquare sense. Furthermore, how to reduce the conservativeness of the stability criterion will become the authors' future work.

\section{Acknowledgements}

The third author would like to thank the Alexander-von-Humboldt Foundation for providing the support to this research possible. The authors are also grateful to the anonymous reviewers for their helpful comments.

\section{Funding}

This work was supported by the National Natural Science Foundation of China under [grant number 61273120].

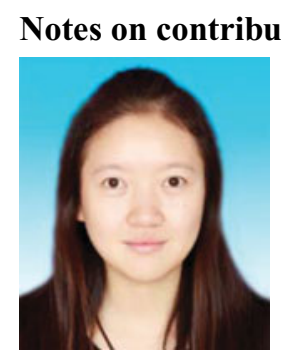

Zhaoxia Duan was born in Sichuan Province, China, in 1989. She received her BS degree in Automation from Nanjing University of Science and Technology (NUST), Nanjing, China, in 2011. Now she is pursuing a $\mathrm{PhD}$ degree in Control Theory and Control Engineering from NUST, Nanjing, China. Her current research interests include robust control and filtering, switched systems, two-dimensional systems and nonlinear systems.

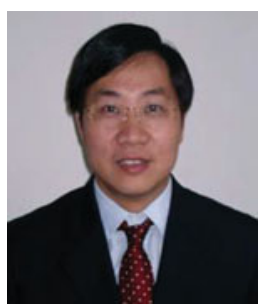

Zhengrong Xiang received his $\mathrm{PhD}$ degree in Control Theory and Control Engineering at Nanjing University of Science and Technology, Nanjing, China, in 1998. Since 1998, he has been faculty member and he is currently full professor at Nanjing University of Science and Technology. He was appointed as Lecturer in 1998 and Associate Professor in 2001 at Nanjing University of Science and Technology. He is a member of the IEEE, member of the Chinese Association for Artificial Intelligence. His main research interests include switched systems, nonlinear control, robust control and networked control systems.

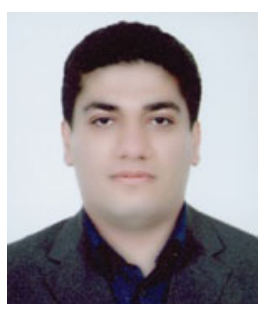

Hamid Reza Karimi is a Professor in Control Systems at the Faculty of Engineering and Science of the University of Agder in Norway. His research interests are in the areas of nonlinear systems, networked control systems, robust control/filter design, timedelay systems, wavelets and vibration control of flexible structures with an emphasis on applications in engineering. He is a 
senior member of IEEE and serves as chairman of the IEEE chapter on control systems at IEEE Norway section. He is also serving as an editorial board member for some international journals, such as Mechatronics, Neurocomputing, Information Sciences, Asian Journal of Control, Journal of Franklin Institute, Journal of Systems and Control Engineering, and International Journal of Control, Automation and Systems. He is a member of IEEE Technical Committee on Systems with Uncertainty, IFAC Technical Committee on Robust Control and IFAC Technical Committee on Automotive Control.

\section{References}

Bisiacco, M. (1995), 'New Results in 2D Optimal Control Theory', Multidimensional Systems and Signal Processing, 6, 189-222.

Bouhtouri, A.E., Hinrichsen, D., and Pritchard, A.J. (1999), ' $H_{\infty}$ Type Control for Discrete-Time Stochastic Systems', International Journal of Robust Nonlinear Control, 9, 923-948.

Chen, S.(2010), 'Delay-Dependent Stability for 2D Systems with Delays in the Roesser Model', in Proceedings of American Control Conference, Baltimore, 30 June-July 02, pp. 3470 3474.

Dhawan, A. (2012), 'Non-Fragile Controller Design for 2-D Discrete Uncertain Systems Described by the Roesser Model', Journal of Signal and Information Processing, 3, 248-251.

Dragan, V., Halanay, A., and Morozan, T. (1992), 'Optimal Stabilizing Compensator for Linear Systems With State Dependent Noise', Stochastic Analysis and Applications, 10, 1131-1143.

$\mathrm{Du}, \mathrm{C}$., and Xie, L. (2002), $H_{\infty}$ Control and Filtering of TwoDimensional Systems (Vol. 278), Berlin: Springer.

Fornasini, E., and Marchesini, G. (1976), 'State-Space Realization Theory of Two-Dimensional Filters', IEEE Transactions on Automatic Control, 21, 484-492.

Gao, Z.Y., and Ahmed, N.U. (1986), 'Stabilizability of Certain Stochastic Systems', International Journal of System Science, 17, 1175-1185.

Gao, Z.Y., and Ahmed, N.U. (1987), 'Feedback Stabilizability of Non-Linear Stochastic Systems With State Dependent Noise', International Journal of Control, 45, 729-737.

Gao, H., Lam, J., Wang, C., and Xu, S. (2004), 'Robust $H_{\infty}$ Filtering for 2D Stochastic Systems', Circuits, Systems, and Signal Processing, 23, 479-505.

Gao, H., Lam, J., Xu, S., and Wang, C. (2005), 'Stability and Stabilization of Uncertain 2D Discrete Systems with Stochastic Perturbation', Multidimensional Systems and Signal Processing, 16, 85-106.

He, Y., Wang, Q., Lin, C., and Wu, M. (2007), 'Delay-RangeDependent Stability for Systems With Time-Varying Delay', Automatica, 43, 371-376.

Hinamoto, T. (1997), 'Stability of 2-D Discrete Systems Described by the Fornasini-Marchesini Second Model', IEEE Transactions on Circuits and Systems I: Fundamental Theory and Applications, 44, 254-257.

Hu, J., Wang, Z., Gao, H., and Stergioulas L.K. (2012), 'Robust Sliding Mode Control for Discrete Stochastic Systems With Mixed Time Delays, Randomly Occurring Uncertainties, and Randomly Occurring Nonlinearities', IEEE Transactions on Industrial Electronics, 59, 3008-3015.

Huang, S., and Xiang, Z. (2013), 'Delay-Dependent Robust $H_{\infty}$ Control for 2D Discrete Nonlinear Systems with State Delays', Multidimensional Systems and Signal Processing, DOI: $10.1007 / \mathrm{s} 11045-013-0230-\mathrm{y}$.

Kaczorek, T. (1985), Two-Dimensional Linear Systems, Berlin: Springer.
Liang, J., Wang, Z., and Liu, X. (2008), 'Exponential Synchronization of Stochastic Delayed Discrete-Time Complex Networks', Nonlinear Dynamics, 53, 153-165.

Liang, J., Wang, Z., and Liu, X. (2012), 'Robust State Estimation for Two-Dimensional Stochastic Time-Delay Systems With Missing Measurements and Sensor Saturation', Multidimensional Systems and Signal Processing, DOI: 10.1007/s11045012-0197-0.

Liu, Y., Wang, Z., and Wang, W. (2011), 'Reliable Filtering for Discrete Time-Delay Systems With Randomly Occurred Nonlinearities Via Delay-Partitioning Method', Signal Processing, 91, 713-727.

Pakshin, P., Galkowski, K., and Rogers, E. (2011), 'Stability and Stabilization of Systems Modeled by 2D Nonlinear Stochastic Roesser Models', The 7th International Workshop on Multidimensional Systems, 5-7 September, pp. 1-5.

Paszke, W., Lam, J., Galkowski, K., Xu, S., and Lin, Z. (2004), 'Robust Stability and Stabilisation of 2D Discrete State-Delayed Systems', Systems \& Control Letters, 51, 277-291.

Sebek, M. (1993), ' $H_{\infty}$ Problem of 2D Systems', in Proceedings of European Control Conference, pp. 1476-1479.

Shen, B., Wang, Z., Shu, H., and Wei, G. (2010), 'Robust $H_{\infty}$ Finite-Horizon Filtering With Randomly Occurred Nonlinearities and Quantization Effects', Automatica, 46, 1743-1751.

Wan, X., and Fang, H. (2013), 'Fault Detection for DiscreteTime Networked Nonlinear Systems with Incomplete Measurements', International Journal of Systems Science, 44, 2068-2081.

Wang, Z., Liu, Y., and Liu, X. (2008), ' $H_{\infty}$ Filtering for Uncertain Stochastic Time-Delay Systems With Sector-Bounded Nonlinearities', Automatica, 44, 1268-1277.

Wang, Z., Liu, Y., Guo, L., and Liu, X. (2010), 'A Note on Control of a Class of Discrete-Time Stochastic Systems with Distributed Delays and Nonlinear Disturbances', Automatica, 46, 543-548.

Wang, Y., Wang, Z., and Liang, J. (2008), 'A Delay Fractioning Approach to Global Synchronization of Delayed Complex Networks with Stochastic Disturbances', Physics Letters A, 372, 6066-6073.

Wang, Y., Wang, Z., and Liang, J. (2009), 'Global Synchronization for Delayed Complex Networks with Randomly Occurring Nonlinearities and Multiple Stochastic Disturbances'. Journal of Physics A: Mathematical and Theoretical, 42, 1751-8113.

Wang, Z., Yang, F., and Liu, X. (2006), 'Robust Filtering for Systems with Stochastic Non-Linearities and Deterministic Uncertainties', Proceedings of the Institution of Mechanical Engineers, Part I: Journal of Systems and Control Engineering, 220, 171-182.

Wei G., Wang Z., and Shen B. (2013), 'Probability-Dependent Gain-Scheduled Control for Discrete Stochastic Delayed Systems With Randomly Occurring Nonlinearities', International Journal of Robust and Nonlinear Control, 23, 815-826.

Xie, L. (1996), 'Output Feedback $H_{\infty}$ Control of Systems with Parameter Uncertainty', International Journal of Control, $63,741-750$

Xu, S., and Lam J. (2005), 'Improved Delay-Dependent Stability Criteria for Time-Delay Systems', IEEE Transactions on Automatic Control, 50, 384-387.

$\mathrm{Xu}, \mathrm{S}$., Lam, J., and Zou, Y. (2005), 'Improved Conditions on Delay-Dependent Robust Stability and Stabilization of Uncertain Discrete Time-Delay Systems', Asian Journal of Control, 7, 344-348.

Xu, J.M., and Yu, L. (2006), ' $H_{\infty}$ Control of 2D Discrete State Delay Systems', International Journal of Control, Automation, and Systems, 4, 516-523. 
Xu, H., Zou, Y., Lu, J., and Xu, S. (2005), 'Robust $H_{\infty}$ Control for a Class of Uncertain Nonlinear Two-Dimensional Systems with State Delay', Journal of the Franklin Institute, 342, 877-891.

Yang, F., Wang, Z., and Ho, D.W.C. (2006), 'Robust Mixed $\mathrm{H}_{2} / \mathrm{H}_{\infty}$ Control for a Class of Nonlinear Stochastic Systems', IEE Proceedings of Control Theory and Applications, 153, $175-184$.

Yao, J., and Wang, W. (2010), 'Robust Stabilization of 2D StateDelayed Systems with Stochastic Perturbation', in The 11th
International Conference of Control, Automation, Robotics and Vision, Singapore, 7-10December, pp. 1951-1956.

Yu, W., Cao, J., and Lü, J. (2008), 'Global Synchronization of Linearly Hybrid Coupled Networks With Time-Varying Delay', SIAM Journal on Applied Dynamical Systems, 7, 108-133.

Zhang, G., Wang, T., Li T., and Fei S. (2013), 'Delay-DerivativeDependent Stability Criterion for Neural Networks With Probabilistic Time-Varying Delay', International Journal of Systems Science, 44, 2140-2151. 